(C) 1981. The Genetical Society of Great Britain

\title{
SEXUAL SELECTION AT A MULTIALLELIC LOCUS WITH COMPLETE OR PARTIAL DOMINANCE*
}

\author{
SAMUEL KARLIN and PETER O'DONALD \\ Department of Mathematics, Stanford University, Stanford, California 943n5; \\ Department of Genetics, University of Cambridge CB2 3EH,England
}

Received 13.i.81

\section{SUMMARY}

In this paper we analyse three general models of sexual selection. In one of the models, an ordered series of multiple alleles determines a series of distinct phenotypes in a dominance hierarchy, each phenotype being dominant to all phenotypes below it in the hierarchy. In another model, some alleles determine an ordered hierarchy of dominant phenotypes; others are co-dominant, each genotype producing a separate phenotype except when in combination with one of the dominant alleles. In the third model, two groups of alleles form two separate dominance hierarchies with co-dominance between alleles from each group. In the model with ordered dominance, a unique, globally stable equilibrium is attained at which the frequencies of the phenotypes are exactly proportional to their rates of preferential mating. In the model with dominance and co-dominance, a unique, globally stable equilibrium is also attained. The gene frequencies at equilibrium are given by hybrid expressions combining the frequencies for complete dominance with those for complete co-dominance. An explicit solution is not found when the alleles form two separate dominance hierarchies; but a unique, globally stable equilibrium exists from which the equilibrium frequencies can be obtained by an iterative method. We also analyse some special models with a limited number of alleles and different dominance and co-dominance relationships. In a model with inclusion relationships between the alleles, an allele giving rise to one sexually selected dominant phenotype may also be included with another allele to give rise to a separate sexually selected phenotype. Thus the genotypes $A_{1} A_{1}, A_{1} A_{2}$ and $A_{1} A_{3}$ may represent one sexually selected phenotype; the genotypes $A_{1} A_{1}, A_{1} A_{2}, A_{1} A_{3}$, $A_{2} A_{2}$ and $A_{2} A_{3}$ may represent another sexually selected phenotype. This is a model of how sexual selection might operate in favour of increasing expression of a character: some females may respond to males with either the alleles $A_{1}$ or $A_{2}$; other females may only respond to males with the more extreme phenotype determined by the allele $A_{1}$. At equilibrium, the allele $A_{2}$ will have been eliminated.

\section{INTRODUCTION}

POLYMORPHISMS in colour patterns are often determined by a series of multiple alleles that can be arranged in a dominance hierarchy in which one allele is dominant or partially dominant to all the others below it in the hierarchy. The following examples represent polymorphisms of this sort: the mimics of the butterflies Papilio dardanus and Papilio memnon (Sheppard, 1961; Clarke et al., 1968), and Hypolimnas misippus (Smith, 1973); melanic and non-melanic forms of the moth Biston betularia (Kettlewell, 1973), of the feral pigeon (Murton et al., 1973), and of the ladybird beetle Adalia bipunctata (Hodek, 1973; Muggleton, 1978, 1979); shell colours of the snail Cepaea memoralis (Cain et al., 1960), and colours and

* Supported in part by NIH 5R01 GM10452-17, NSF MCS79-24310 and SRC GR/B30807. 
shell banding patterns in many other snails. The alleles may often be more or less completely dominant in their effects, but some of the heterozygotes may be distinct phenotypes, as for example in Papilio dardanus and Adalia bipunctata.

Some of these examples of polymorphisms show the effects of preferential mating. Melanic and non-melanic morphs of the feral pigeon assort negatively at mating: wild-types prefer the melanic morphs blue checker and dark blue checker (Murton et al., 1973). This observation suggests that wild-type females may prefer to mate with the melanic males. Davis and O'Donald (1976) estimated the levels of female preference by fitting a model of negative assortative mating to the data. Burns (1966) showed that sexual selection may operate between morphs of Hypolimnas misippus. Muggleton (1979) and O'Donald and Muggleton (1979) showed that melanic and non-melanic morphs of Adalia bipunctata were the objects of strong sexual selection. In Adalia, the polymorphism consists of a series of morphs that can be arranged in a series of increasing melanism. The non-melanic morph, which is red with two black spots, is recessive to all the melanic morphs. If the melanics are all linked together in a single class, frequency-dependent sexual selection can then be seen to operate between the melanics and non-melanics: a mating advantage is gained by the rarer phenotype. Preferential mating of melanic and non-melanic phenotypes would produce this frequency-dependence in mating advantage (O'Donald, 1980). The different colours of the male ruff (Philomachus pugnax) and guppy (Poecilia reticulata) are certainly selected sexually. The different forms of guppies do indeed show the "rare male" effect characteristic of sexual selection by female choice (Farr, 1977). There are other examples of polymorphisms that are probably determined by a series of dominant or partly dominant alleles and maintained by sexual selection.

In theoretical studies of sexual selection for dominant and recessive phenotypes, models have been analysed in which there are only two phenotypes, one dominant to the other (O'Donald, 1963, 1973, 1977, 1978, 1980; Karlin, 1978; Karlin and Raper, 1979; Karlin and Farkash, 1978). In this paper we analyse more general models with multiple alleles. These models are directly applicable to some of the polymorphisms already mentioned. In particular, the polymorphism in Adalia bipunctata appears to be maintained by preferential mating of phenotypes determined by a series of at least twelve alleles with a partial dominance hierarchy in which some heterozygotes can be recognised. As well as the general model, in which each allele is dominant to all alleles below it in the hierarchy, we also analyse models with only partial dominance. In models with partial dominance, some heterozygotes are distinct for any homozygote and thus may be subject to separate preferences. These models will certainly be applicable to specific polymorphisms like that of Adalia in which dominance may not be complete for all alleles.

In an alternative model (Section 6) with partial dominance, and inclusion relationships, females are more responsive to the more extreme phenotype. Thus, in Adalia bipunctata, some females may respond to all melanic phenotypes; others may only respond to the darker or darkest melanics. This mating behaviour would result if the darker melanics provided the greater stimulus so that more females preferred them. 


\section{THE COMPLETE DOMINANCE ORDERING MODEL}

In the sexual selection model for a one locus diallelic trait where allele $A$ is dominant to allelele $a$, there are two phenotypic classes $\bar{A}=\{A A, A a\}$ and $\{a a\}$, with preferential mating rates $\alpha$ and $\gamma$. The rates $\alpha$ and $\gamma$ refer to the dominant and recessive types specifying the fraction of females, independent of their genotype, that choose males of the respective types. We assume: all females are fertilised, males are polygamous; population sizes are large; and there is a 1:1 sex ratio (cf. O'Donald, 1977; Karlin, 1978). In this framework there exists a unique globally attracting polymorphism at Hardy-Weinberg frequencies with

$$
\begin{aligned}
& p_{A}=\text { frequency of allele } A=1-\sqrt{\frac{\gamma}{\alpha+\gamma}} \\
& p_{a}=\text { frequency of allele } a=\sqrt{\frac{\gamma}{\alpha+\gamma}} .
\end{aligned}
$$

In this study we investigate the extension of this model to a dominance hierarchy of $n$-alleles. We concentrate first on the case of a complete ordering of dominance and in the following sections some formulations of partial and mixed dominance relationships are considered. The interest and relevance of these models is discussed in the introduction. Some interpretations and implications of our results are provided in the concluding section.

Consider a population of a trait involving $n$-alleles subject to the dominance ordering

$$
A_{1}<A_{2}<A_{3}<\cdots<A_{n}
$$

representing $n$ phenotypes, the phenotypic expression depending on the dominant allele. Specifically, the phenotype classes consist of the genotypes as shown:

$$
\mathscr{P}_{k}=\left\{A_{k} A_{k}, A_{k} A_{k-1}, \ldots, A_{k} A_{1}\right\} \quad k=1,2, \ldots, n .
$$

Let the mating preference rate for $\mathscr{P}_{k}$ be $\alpha_{k}$, that is, a fraction $\alpha_{k}$, $k=1,2, \ldots, n$, of all females select a male of phenotype $\mathscr{P}_{k}$ and the remaining fraction of the female population $1-\sum_{i=1}^{n} \alpha_{i}$ exercise no mating preference, $i . e$., they choose mates at random from the male population.

It is convenient to introduce the ordered genotype frequencies

$$
u_{i j}=\text { freq }\left(A_{i} A_{i}\right) \text { in the female population at the zygote stage, }
$$

where the first (second) allele of the genotype $A_{i}\left(A_{j}\right)$ corresponds to the maternal (paternal) gamete contribution of the preceding generation. Of course, $u_{i j}=u_{j i}$. At the zygote state, the equal sex ratio gives rise to the same genotypic frequencies in males and females.

To ease the later developments we set the notation for the allele frequencies and phenotype frequencies to correspond to (4). Thus

$$
\begin{aligned}
p_{i} & =\operatorname{freq}\left(A_{i}\right) \\
& =\sum_{r=1}^{n}\left(\frac{u_{i r}+u_{r i}}{2}\right) \quad i=1,2, \ldots, n
\end{aligned}
$$


and

$$
\begin{aligned}
\pi_{k} & =\text { frequency of phenotype } \mathscr{P}_{k} \\
& =u_{k k}+\sum_{r=1}^{k-1}\left(u_{k r}+u_{r k}\right) .
\end{aligned}
$$

In the analyses to follow we rely heavily on the fact established by Karlin (1978, section 7) that for all models of sexual selection in which certain subpopulations of females exercise preferences towards different male phenotypes and which may allow a host of ecological, physiological or behavioural adaptations, the equilibrium frequency configurations are always in Hardy-Weinberg proportions. In particular, this result applies to both monogamous and polygamous bond formation, variation of female response to male behavioural patterns, intensity of male courtship, and natural selection forces operating on the male. The Hardy-Weinberg property for equilibrium states is generally not valid under assortative mating or for regular inbreeding systems.

The recursion relations for the general model reduce to (see Karlin, 1978, equation 7.9)

$$
u_{i j}^{\prime}=\frac{1}{2}\left[p_{i} f_{i}+p_{i} f_{i}\right]
$$

where

$$
f_{i}=f_{i i}+\frac{1}{2} \sum_{\substack{r=1 \\ r \neq i}}^{n} f_{i r}
$$

and $f_{i j}$ is the effective male frequency of genotype $A_{i} A_{j}$ participating in or responding to sexual selection and random mating propensities and associated behavioural requirements.

In the special case of the $n$-allele complete dominance model (see (2)), we have

$$
f_{i j}=\alpha_{i} \frac{u_{i j}+u_{i i}}{\pi_{i}}+\left(1-\sum_{r=1}^{n} \alpha_{r}\right)\left(u_{i j}+u_{i i}\right) \quad i<j .
$$

Indeed, a proportion $\alpha_{j}$ of females have prescribed mates in phenotype class $\mathscr{P}_{j}$. The quantity $\left(u_{i j}+u_{j i}\right) / \pi_{j}$ is the fraction of males carrying genotype $A_{i} A_{i}$ in the phenotype class $\mathscr{P}_{j}$.

The fraction $1-\sum_{r=1}^{n} \alpha_{r}$ of females choose mates at random from the male population at large.

The recursion equations connecting allele frequencies over successive generations operating on (7) takes the form

$$
p_{i}^{\prime}=\frac{1}{2}\left[p_{i}+f_{i}\right]
$$

where $f_{i}$ is defined in (8). We substitute from (9) to get

$$
\begin{array}{r}
p_{i}^{\prime}=\frac{1}{2}\left[p_{i}+\alpha_{i}\left(\sum_{i=1}^{j}\left(\frac{u_{i j}+u_{i j}}{2}\right)\right) / \pi_{i}+\sum_{r=j+1}^{n} \alpha_{r}\left(\frac{u_{r j}+u_{i r}}{2 \pi_{r}}\right) p_{i}+\left(1-\sum_{i=1}^{n} \alpha_{i}\right) p_{i}\right] \\
j=1,2, \ldots, n .
\end{array}
$$


At an equilibrium, $u_{i j}^{*}=2 p_{i}^{*} p_{i}^{*}, i \neq j, u_{i i}^{*}=p_{i}^{* 2}$ with all $p_{i}^{*}>0$, the equation (11) with some simplification reduces to

$$
L p_{i}^{*}=\alpha_{j} \frac{K_{i}^{*}}{K_{i}^{*}+K_{i-1}^{*}}+p_{i}^{*} \sum_{r=j+1}^{n} \frac{\alpha_{r}}{K_{r}^{*}+K_{r-1}^{*}} \quad j=1,2, \ldots, n,
$$

where $K_{i}^{*}=\sum_{i=1}^{i} p_{i}^{*}$ and $K_{n}^{*}=1, L=\sum_{i=1}^{n} \alpha_{i}$. Solving by backward induction, we obtain

$$
L p_{n}^{*}=\frac{\alpha_{n}}{K_{n}^{*}+K_{n-1}^{*}}=\frac{\alpha_{n}}{2-p_{n}^{*}}
$$

and therefore

$$
\left(1-p_{n}^{*}\right)^{2}=1-\frac{\alpha_{n}}{L} \quad \text { or } \quad p_{n}^{*}=1-\sqrt{\sum_{i=1}^{n-1} \frac{\alpha_{i}}{L}} .
$$

Using (14) in (12) for the equation $j=n-1$ gives

$$
L p_{n-1}^{*}=\alpha_{n-1} \frac{K_{n-1}^{*}}{K_{n-1}^{*}+K_{n-1}^{*}}+p_{n-1}^{*} L p_{n}^{*}
$$

which implies $L p_{n-1}^{*}=\alpha_{n-1} /\left(K_{n-1}^{*}+K_{n-2}^{*}\right)$ and inductively

$$
L p_{k}^{*}=\frac{\alpha_{k}}{K_{k}^{*}+K_{k-2}^{*}} \quad k=1,2, \ldots, n .
$$

The equations can be solved recursively yielding

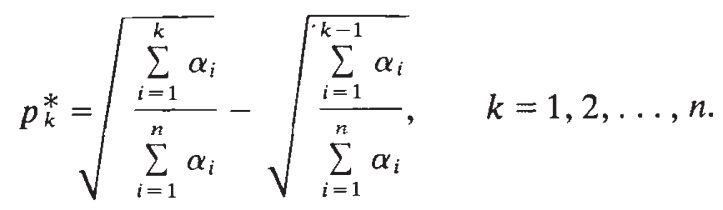

Moreover at equilibrium, see (15),

$$
\left(K_{r}^{*}+K_{r-1}^{*}\right) p_{r}^{*}=\pi_{r}^{*}=\frac{\alpha_{r}}{\sum_{i=1}^{n} \alpha_{i}}
$$

which shows that the phenotype equilibrium frequencies are exactly proportional to the preferential mating rates for the corresponding phenotypes.

The allele frequency levels are marginally of the order of the square root of the $\left\{\alpha_{k}\right\}$ as exhibited in (16). In particular, when $\alpha_{1}=\alpha_{2}=\cdots=\alpha_{n}$ then $p_{k}^{*}=\sqrt{k / n}-\sqrt{(k-1) / n}, k=1,2, \ldots, n$. Thus, for $\alpha_{i}$ equal, $p_{1}^{*}>$ $p_{2}^{*}>\cdots>p_{n}^{*}$.

We may inquire what preference rates correspond to equal equilibrium allele frequencies. This requires the approximate determination $\alpha_{k} \sim$ $c /(k+1)^{2}$.

Observe from (16) that in the case $\alpha_{k_{0}}=0$ (no special preference for allele $A_{k_{0}}$ ) then $p_{k_{0}}^{*}=0$. This result may have been expected.

\section{A MIXED MODEL OF DOMINANCE AND CODOMINANCE}

Consider first the sexual selection model of $n$ codominant alleles where each genotype engenders a distinct phenotype. If the mating preference 
rate for $A_{i} A_{j}$ males is $\alpha_{i j}$, the unique stable equilibrium has allele frequencies

$$
p_{i}^{*}=p^{*}\left(A_{i}\right)=\frac{\alpha_{i i}+\frac{1}{2} \sum_{i=1, j \neq 1}^{n} \alpha_{i j}}{\sum_{k, l} \alpha_{k l}}
$$

with corresponding genotype frequencies determined from these allele frequencies in Hardy-Weinberg ratios.

Consider next the hierarchical structure

$$
A_{n}>A_{n-1}>\cdots>A_{k_{0}}>\left\{\begin{array}{c}
A_{k_{0}}-1 \\
\vdots \\
A_{2} \\
A_{1}
\end{array}\right\}
$$

signifying that $A_{m}, m=n, n-1, \ldots, k_{0}$, are each alleles dominant to alleles of lower indices forming the phenotype grouping

$$
\begin{aligned}
& \mathscr{P}_{n}=\left\{A_{n} A_{n}, A_{n} A_{n-1}, \ldots, A_{n} A_{1}\right\} \\
& \mathscr{P}_{n-1}=\left\{A_{n-1} A_{n-1}, A_{n-1} A_{n-2}, \ldots, A_{n-1} A_{1}\right\} \\
& \vdots \\
& \mathscr{P}_{k_{0}}=\left\{A_{k_{0}} A_{k_{0}}, A_{k_{0}} A_{k_{0}-1}, \ldots, A_{k_{0}} A_{1}\right\}
\end{aligned}
$$

while the other genotypes

$$
\left\{A_{r} A_{s}\right\} \quad 1 \leqq r \leqq s \leqq k_{0}-1
$$

express distinguishable phenotypes. Let

$$
\left\{\alpha_{n}, \alpha_{n-1}, \ldots, \alpha_{k_{0}}, \gamma_{r s} \quad 1 \leqq r \leqq s \leqq k_{0}-1\right\}
$$

be the preferential mating rates for the various phenotypes. Extending the analysis of the previous section we can establish the following result. The unique stable equilibrium allele frequencies for the phenotype classification of (18) with sexual selection parameters (19) are explicitly given by

$$
\begin{aligned}
p_{n}^{*}= & 1-\sqrt{\frac{L-\alpha_{n}}{L}} \\
p_{i}^{*}= & \sqrt{\frac{L-\alpha_{n}-\alpha_{n-1}-\cdots-\alpha_{i+1}}{L}} \\
& -\sqrt{\frac{L-\alpha_{n}-\alpha_{n-1}-\cdots-\alpha_{i}}{L}}, \quad k_{0} \leqq i \leqq n-1
\end{aligned}
$$

with $L=\sum_{i=k_{0}}^{n} \alpha_{i}+\sum_{r, s=1}^{k_{0}-1} \gamma_{r, s}$, while

$$
p_{i}^{*}=\left(\sqrt{\frac{\sum_{r, s=1}^{k_{0}-1} \gamma_{r s}}{L}}\right)\left(\frac{\gamma_{i i}+\sum_{r=1, r \neq i}^{1} \sum_{r, s}^{k_{0}-1} \gamma_{r i}}{\sum_{r, s}^{k_{0}-1} \gamma_{r s}}\right), \quad i=1,2, \ldots, k_{0}
$$


The genotype frequencies are determined from $\left\{p_{i}^{*}\right\}$ in Hardy-Weinberg proportions.

In comparing (20) with (16) we find that this model appears to be a hybrid version of the models with complete dominance and with complete codominance. This result is not found when partial dominance patterns do not factorise so simply.

\section{Models With SEPARATE SERIES OF ALlelic DOMINANCE ORDERINGS}

Consider a locus with $n+m$ alleles composed of two all-allelic series

and

$$
A_{1}<A_{2}<A_{3}<\cdots<A_{n}
$$

$$
B_{1}<B_{2}<B_{3}<\cdots<B_{m}
$$

where the notation indicates that the $A$ alleles form a dominance hierarchy as in section 2 of $n$ phenotype classes

$$
\mathscr{P}_{k}(A)=\left\{A_{k} A_{k}, A_{k} A_{k-1}, \ldots, A_{k} A_{1}\right\}, \quad k=1,2, \ldots, n
$$

and the $B$ alleles form a separate dominance ordering

$$
\mathscr{P}_{l}(B)=\left\{B_{l} B_{l}, B_{l} B_{l-1}, \ldots, B_{l} B_{1}\right\}, \quad l=1,2, \ldots, m .
$$

The genotypes $\left\{A_{i} B_{j}\right\}$ are assumed to be codominant in phenotypic expression. Suppose the preferential mating rates are

$$
\alpha_{1}, \alpha_{2}, \ldots, \alpha_{n} \text { with respect to the phenotype classes } \mathscr{P}_{k}(\boldsymbol{A}),
$$

and

$\beta_{1}, \beta_{2}, \ldots, \beta_{m}$ for the phenotype classes $\mathscr{P}_{l}(B)$.

Let the preference rates for the phenotype = genotypes

$$
\left\{A_{k} B_{l}\right\} \text { be } \gamma_{k l}, \quad k=1,2, \ldots, n, \quad l=1,2, \ldots, m .
$$

Using the fact that all equilibria exhibit Hardy-Weinberg form, and the general recursion relations $(10)$, we can establish the existence of a unique stable equilibrium. Let $p_{k}^{*}, k=1,2, \ldots, n, q_{i}^{*}, l=1,2, \ldots, m$ be the equilibrium allele frequencies.

The individual $p_{i}^{*}$ and $q_{i}^{*}$ satisfy the equations

$$
\begin{aligned}
& L p_{i}^{*}=\alpha_{i} \frac{K_{i}^{*}}{K_{i}^{*}+K_{i-1}^{*}}+\left(\sum_{l=i+1}^{n} \frac{\alpha_{l}}{K_{l}^{*}+K_{l-1}^{*}}\right) p_{i}^{*}+\frac{1}{2} \sum_{j=1}^{m} \gamma_{i j}, \quad i=1,2, \ldots, n \\
& L q_{j}^{*}=\beta_{j} \frac{L_{j}^{*}}{L_{j}^{*}+L_{j-1}^{*}}+\left(\sum_{l=j+1}^{n} \frac{\beta_{l}}{L_{l}^{*}+L_{l-1}^{*}}\right) q_{j}^{*}+\frac{1}{2} \sum_{i=1}^{n} \gamma_{i j}, \quad j=1,2, \ldots, m,
\end{aligned}
$$

where

$$
K_{i}^{*}=\sum_{\lambda=n-i+1}^{n} p_{\lambda}^{*} \quad \text { and } \quad L_{j}^{*}=\sum_{\mu=m-j+1}^{m} q_{\mu}^{*}
$$

following the analysis of (12). 
We obtain explicitly

and

$$
K_{n}^{*}=\sum_{i=1}^{n} p_{i}^{*}=\frac{L_{A}}{L}=\frac{\sum_{i=1}^{n} \alpha_{i}+{ }_{2}^{1} \sum_{k, l} \gamma_{k l}}{\sum_{i=1}^{n} \alpha_{i}+\sum_{j=1}^{m} \beta_{j}+\sum_{k, l} \gamma_{k l}}
$$

$$
L_{m}^{*}=\sum_{j=1}^{m} q_{j}^{*}=\frac{L_{B}}{L}=1-\frac{L_{A}}{L}
$$

These formulas show that the separate conglomerates of the $A$ and $B$ series of alleles at equilibrium behave as two simple codominant alleles.

We validate the relation (27) as follows. Adding the equations of (24) gives

$$
\begin{aligned}
L K_{n}^{*} & =\sum_{i=1}^{n} \alpha_{i} \frac{K_{i}^{*}}{K_{i}^{*}+K_{i-1}^{*}}+\sum_{k=1}^{n}\left(\sum_{l=k+1}^{n} \frac{\alpha_{l}}{K_{l}^{*}+K_{l-1}^{*}}\right) p_{k}^{*}+\frac{1}{2} \sum_{k, l} \gamma_{k l} \\
& =\sum_{i=1}^{n} \alpha_{i}+{ }_{2}^{1} \sum_{k, l} \gamma_{k l}=L_{A} .
\end{aligned}
$$

The equation (24) for $k=n$ in view of (27) reduces to

$$
L p_{n}^{*}=\alpha_{n} \frac{K_{n}^{*}}{2 K_{n}^{*}-p_{n}^{*}}+\frac{1}{2} \sum_{j=1}^{m} \gamma_{n j}
$$

with $K_{n}^{*}=L_{A} / L$. This quadratic in $p_{n}^{*}=p$, namely

$$
L p^{2}-\left(2 K_{n}^{*} L+\frac{1}{2} \sum_{j=1}^{m} \gamma_{n j}\right) p=-\left(\alpha_{n}+\sum_{j=1}^{m} \gamma_{n j}\right) K_{n}^{*}
$$

admits a unique positive solution. Once $p_{n}^{*}$ is determined, then $K_{n-1}^{*}$ is known and the equation (24) for $k=n-1$ can be used to solve for $p_{n-1}^{*}$ again from a quadratic equation. This procedure can be iterated to calculate all $p_{k}^{*}$. In a similar manner the values $q_{i}^{*}$ are uniquely determined.

The results can be generalized to a multiallelic model comprised of $r$ dominance series. Indeed, suppose the $i$ th allelic group obeys a dominance ordering

$$
A_{1}^{(i)}<A_{2}^{(i)}<\cdots<A_{n_{i}}^{(i)} \quad i=1,2, \ldots, r
$$

with codominance phenotypic expression between alleles of different series. Let the preference rates for the dominance class of $A_{\lambda}^{(i)}$ be

$$
\alpha_{\lambda}^{(i)} \quad \lambda=1,2, \ldots, n_{i} ; \quad i=1,2, \ldots, r
$$

and $\gamma_{k, l}^{(i, i)}$ the preference mating rate for genotype $\left\{A_{k}^{(i)} A_{l}^{(i)}\right\}$ for $i \neq j$.

For the foregoing sexual selection random mating model there exists a unique stable Hardy-Weinberg equilibrium array with allele frequencies $\hat{p}_{\lambda}^{(i)}=$ freq $\left(A_{\lambda}^{(i)}\right)$ constrained to satisfy

$$
\hat{K}^{(i)}=\sum_{\lambda=1}^{n_{i}} \hat{p}_{\lambda}^{(i)}=\frac{\sum_{\lambda=1}^{n_{i}} \alpha_{\lambda}^{(i)}+\frac{1}{2} \sum_{k, l, j} \gamma_{k, l}^{(i, j)}}{\sum_{i, \lambda} \alpha_{\lambda}^{(i)}+\sum_{i, j, k, l} \gamma_{k, l}^{(i, j)}} .
$$


The individual $\hat{p}_{\lambda}^{(i)}$ can be computed recursively as the roots of appropriate quadratic polynomials.

\section{SoME SPECIAL MODELS}

Consider the three allele model with phenotypes

$\begin{array}{lccccc} & \left\{A_{1} A_{1}, A_{1} A_{2}\right\} & \left\{A_{1} A_{3}\right\} & \left\{A_{2} A_{2}\right\} & \left\{A_{2} A_{3}\right\} & \left\{A_{3} A_{3}\right\} \\ \text { preferential } & \alpha & \gamma_{1} & \beta & \gamma_{2} & \gamma_{3}\end{array}$

There exists a unique stable Hardy-Weinberg equilibrium and the allele frequencies can be explicitly determined as solutions of appropriate quadratic equations (see below).

In fact, the model (30) has $A_{1}$ dominant to $A_{2}$ and all other genotypes codominants. The equilibrium equations are

$$
\begin{aligned}
& p_{3}=\gamma_{3}+\frac{\gamma_{1}+\gamma_{2}}{2}+(1-L) p_{3} \\
& p_{2}=\frac{\alpha p_{2}}{p_{1}+2 p_{2}}+\beta+\frac{\gamma_{2}}{2}+(1-L) p_{2} \\
& p_{1}=\frac{\alpha p_{1}+p_{2}}{p_{1}+2 p_{2}}+\frac{\gamma_{1}}{2}+(1-L) p_{1} .
\end{aligned}
$$

The unique solution (abbreviating $L=\alpha+\beta+\gamma_{1}+\gamma_{2}+\gamma_{3}$ ) has

$$
\begin{aligned}
& p_{3}^{*}=\frac{\gamma_{3}+\left(\gamma_{1}+\gamma_{2}\right) / 2}{L} \\
& p_{2}^{*}=\left(-\frac{\left(\gamma_{1}+\gamma_{2}+\beta\right)}{2}+\sqrt{\left(\frac{\gamma_{1}+\gamma_{2}+\beta}{2}\right)^{2}+2\left(\beta+\gamma_{2}\right)\left(1-p_{3}^{*}\right)} L / 2 L\right. \\
& p_{1}^{*}=1-p_{1}^{*}-p_{2}^{*} .
\end{aligned}
$$

The phenotype grouping of nontransitive dominance relationships

$$
\left\{A_{1} A_{1}, A_{1} A_{2}\right\} \quad\left\{A_{2} A_{2}, A_{2} A_{3}\right\} \quad\left\{A_{1} A_{3}, A_{3} A_{3}\right\}
$$

sexual selection rates

$\alpha$

$\beta$

also admits a globally stable equilibrium.

In contrast, the phenotype grouping

$$
\begin{array}{ccc} 
& \left\{A_{1} A_{1}, A_{2} A_{2}\right\} & \left\{A_{1} A_{2}\right\} \\
\text { mating sexual selection rates } & \alpha & \beta
\end{array}
$$

which cannot be interpreted in terms of a dominance relationship, can involve multiple stable (Hardy-Weinberg) equilibria for certain ranges of the $\alpha, \beta$ parameters (see Karlin and Farkash, 1978, table IV).

It appears that with $n$-alleles with phenotype classes determined by partial dominance relations, a unique stable equilibrium exists subject to sexual selection and random mating forces, but this is not the case with phenotype classes determined by genotype groupings not characterized by dominance ordering. 


\section{Phenotype Classes With inclusion Relationships}

It is illuminating to begin with the following three allele model. Consider three phenotypes

$$
\begin{aligned}
\mathscr{P}_{1}= & \left\{A_{1} A_{1}, A_{1} A_{2}, A_{1} A_{3}\right\}=\text { the dominant class of } A_{1}, \\
\mathscr{P}_{1,2}= & \left\{A_{1} A_{1}, A_{1} A_{2}, A_{1} A_{3}, A_{2} A_{2}, A_{2} A_{3}\right\}=\text { the combined } \\
& \text { dominance group determined by alleles } A_{1} \text { and } A_{2}, \\
\mathscr{P}_{3}= & \left\{A_{3} A_{3}\right\} .
\end{aligned}
$$

Suppose the preferential mating rates for the above phenotypes are $\alpha, \beta$ and $\gamma$, respectively. Thus, a fraction $\alpha$ of females prefers the $\mathscr{P}_{1}$ phenotype, but a different subpopulation, a fraction $\beta$ of females, favours any genotype carrying either the $A_{1}$ or the $A_{2}$ allele. Let $p_{1}, p_{2}$, and $p_{3}$ be the frequencies of the $A_{1}, A_{2}$ and $A_{3}$ alleles, respectively. Appealing to the general sexual selection theory (see especially Karlin, 1978, section 7) the equilibrium equations (since the genotype frequencies are necessarily of Hardy-Weinberg form) reduce to

$$
\begin{aligned}
& L p_{1}=\alpha \frac{1}{p_{1}+2 p_{2}+2 p_{3}}+\beta \frac{p_{1}}{1-p_{3}^{2}} \\
& L p_{2}=\alpha \frac{p_{2}}{1+p_{2}+p_{3}}+\beta \frac{p_{2}}{1-p_{3}^{2}}
\end{aligned}
$$

where $L=\alpha+\beta+\gamma$.

Assuming $p_{2}>0$ and cancelling this factor in (32) yields

$$
L=\alpha \frac{1}{1+p_{2}+p_{3}}+\frac{\beta}{1-p_{3}^{2}} .
$$

Substituting this expression into (32) and simplifying leads to the equation

$$
L\left(1-p_{1}\right)=\frac{\beta}{1-p_{3}^{2}}\left(1-p_{1}\right) .
$$

If $p_{1}<1$, we find that $L=\beta /\left(1-p_{3}^{2}\right)$ which in comparison with (34) for $\alpha>0$ is impossible. The case of $p_{1}=1$ (fixation of only $A_{1} A_{1}$-genotypes) cannot be stable with some initial representation of allele $A_{3}$ when $\gamma>0$. Thus, the only consistent conclusion has $p_{2}=0$, i.e., allele $A_{2}$ cannot be maintained under the sexual selection system of (31) for the phenotype classes $\mathscr{P}_{1}, \mathscr{P}_{1,2}$ and $\mathscr{P}_{3}$. This is, a priori, perhaps surprising in view of the existence of some a priori positive preference for phenotype $\mathscr{P}_{1,2}$, which includes some genotypes involving allele $A_{2}$. The intuition after the fact is that, although some fraction among the females indifferently favour the genotypes carrying allele $A_{2}$ and $A_{1}$, there are additional females only willing to accept matings with the dominance class of $\left\{A_{1}\right\}$ thereby inducing a uniform advantage for allele $A_{1}$ over allele $A_{2}$; concomitantly allele $A_{2}$ cannot be maintained.

The foregoing model suggests a mechanism where the lowest dominant allele $A_{3}$ and the highest dominant allele $A_{1}$ are represented with moderate frequency while the intermediate allele $A_{2}$ barely occurs. Actually, there are such documented examples (e.g. Feral pigeon) where the sexual selec- 
tion process coupled to an approximate dominance pattern provides a mechanism of this $\mathrm{U}$-shaped frequency pattern.

The above example is subsumed by the following general formulation. Consider an $n$ allele sexual selection system of possible alleles $A_{i}$ ( $i=$ $1,2, \ldots, n)$ based on a dominance ordering $A_{1}<A_{2}<\cdots<A_{n}$ which entail the phenotype classes

$$
\left\{A_{1}\right\},\left\{\begin{array}{l}
A_{2} \\
A_{3}
\end{array}\right\},\left\{A_{3}\right\},\left\{\begin{array}{l}
A_{4} \\
A_{5} \\
A_{6}
\end{array}\right\},\left\{A_{6}\right\}, \ldots,\left\{A_{n}\right\}
$$

where the symbols are to be interpreted as follows. The phenotype class $\mathscr{P}_{n}$ consists of all genotypes carrying allele $A_{n}$. The phenotype expression of $\mathscr{P}_{n-1}, \ldots, \mathscr{P}_{6}$ is also characterized in a similar manner;

$$
\left\{\begin{array}{l}
A_{4} \\
A_{5} \\
A_{6}
\end{array}\right\} \approx \mathscr{P}_{4,5,6}
$$

refers to the collection of all genotypes carrying alleles $A_{4}$ and/or $A_{5}$ and/or $A_{6}$, and $\mathscr{P}_{2,3}$ stands for the genotypes involving alleles $A_{2}$ and/or $A_{3}$. Now suppose there is positive a-priori mating preference of some fraction of females for each of the male phenotype classes of (36). Then any stable equilibrium necessarily has $p_{2}^{*}=0$ and $p_{4}^{*}=p_{5}^{*}=0$. Thus, even though there is some mating preference for $\mathscr{P}_{4,5,6}$ and $\mathscr{P}_{2,3}$ the special preference for $\mathscr{P}_{6}$ precludes the existence of alleles $A_{5}$ and $A_{4}$ in evolution. Allele $A_{2}$ also cannot be maintained under the phenotype classification (36).

Biologically, this is a realistic model of how preferences might operate to favour increasing levels of expression of a male characteristic. In this general tendency to express a preference, some females will be more responsive than others. In the model specified by (36) above, for example, the more responsive females may prefer males who possess the alleles $A_{1}$ or $A_{2}$ : they choose to mate with any male who is $A_{1} A_{1}, A_{1} A_{2}, A_{1} A_{3}$, $A_{2} A_{2}, A_{2} A_{3}, A_{3} A_{3}$, etc. But the less responsive females may only respond to males in whom the character is more highly developed: these females choose only the $A_{1} A_{1}, A_{1} A_{2}$ or $A_{1} A_{3}$ males.

\section{REFERENCES}

BURNS, J. M. 1966. Preferential mating versus mimicry: disruptive selection and sex-limited dimorphism in Papilio glaucus. Science, 153, 551-553.

CAIN, A. J., KING, J. M. B., AND SHEPPARD, P. M. 1960. New data on the genetics of polymorphism in the snail Cepaea nemoralis. Genetics, 45, 393-411.

CLARKE, C. A., SHEPPARD, P. M., AND THORNTON, I. W. B. 1968. The genetics of the mimetic butterfly Papilio memnon L. Phil. Trans. Roy. Soc. Lond., 254B, 37-89.

DAVIS, J. W. F., AND O'DONALD. P. 1976. Territory size, breeding time and mating preference in the Arctic Skua. Nature, 260, 774-775.

FARR, J. A. 1977. Male rarity or novelty, female choice behaviour, and sexual selection in the guppy, Poecilia reticulata Peters (Pices: Poeciliidae). Evolution, 31, 162-168.

HODEK, I. 1973. Biology of Coccinellidae. Academia, Prague.

KARLIN, S. 1978. Comparisons of positive assortative mating and sexual selection models. Theor. Pop. Biol., 14, 281-312.

KARLIN, S., AND FARKASH, S. 1978. Analysis of partial assortative mating and sexual selection models for a polygamous species involving a trait based on levels of heterozygosity. Theor. Pop. Biol., 14, 430-445. 
KARLin, S., AND RAPER, J. K. 1979. Sexual selection encounter models. Theor. Pop. Biol., $15,246-256$.

KetTlewell, H. B. D. 1973. The Evolution of Melanism. Clarendon Press, Oxford.

MUGGLeton, J. 1978. Selection against the melanic morphs of Adalia bipunctata (two-spot ladybird): A review and some new data. Heredity, 40, 268-290.

Muggleton, J. 1979. Non-random mating in wild populations of polymorphic Adalia bipunctata. Heredity, 42, 57-65.

MURTON, R. K., WESTWOOD, N. J., AND THEARle, R. J. P. 1973. Polymorphism and the evolution of a continuous breeding season in the pigeon, Columba livia. J. Reprod. Fert., Suppl., 19, 563-577.

O'DONALD, P. 1963. Sexual selection for dominant and recessive genes. Heredity, 18, 451-457.

O'DONALD, P. 1973. Models of sexual and natural selection in polygynous species. Heredity, 31, 145-156.

O'DONAld, P. 1977. Theoretical aspects of sexual selection. Theor. Pop. Biol., 12, 298-334.

O'DONALD, P. 1978. Theoretical aspects of sexual selection: a generalised model of mating behaviour. Theor. Pop. Biol., 13, 226-243.

O'donald, P. 1980. Genetic Models of Sexual Selection. Cambridge University Press, Cambridge.

O'DONALD, P., AND MUGGLeTON, J. 1979. Melanic polymorphism in ladybirds maintained by sexual selection. Heredity, 43, 143-148.

SHEPPARD, P. M. 1961. Recent genetical work on polymorphic Papilios. In Insect Polymorphism, ed. J. S. Kennedy, pp. 20-29, Roy. Ent. Soc., London.

SMITH, D. A. S. 1973. Batesian mimicry between Danaus chrysippus and Hypolimnas misippus (Lepidoptera) in Tanzania. Nature, 242, 129-131. 